\title{
CONVERSOR AC-DC EM MODO CONTÍNUO DE CORRENTE COM COMPENSAÇÃO EM FREQUÊNCIA PARA LÂMPADAS LED DE BAIXO CUSTO E ALTA EFICIÊNCIA
}

\section{CONVERTER AC-DC POWER IN CONTINUOUS MODE WITH COMPENSATION IN FREQUENCY FOR LOW COST LED LAMPS AND HIGH EFFICIENCY}

Maria Eduarda A. Boscoli, Araújo C.M.B., Berci C.D., Guelfi, A. E., Donzelli D

Universidade do Oeste Paulista -UNOESTE, Curso de Engenharia Elétrica, Laboratório de Instrumentação e Eletroeletrônica (IEE), Presidente Prudente, SP. E-mail: dudaboscoli@hotmail.com

RESUMO - Este artigo apresenta uma nova arquitetura de um driver de LEDs de alto brilho, com baixo custo e elevado rendimento. Empregase um conversor do tipo CUK em modo contínuo de corrente com compensação em frequência, para alimentação dos LEDs de alto brilho através da rede elétrica, a partir de $85 \mathrm{~V}$ a $265 \mathrm{~V}$, operando em alta frequência com comutação por Modulação por Largura de Pulso (MLP) e conectado a um filtro ressonante LC série para alimentação da carga com proteção contra surto de tensão na entrada. Foram utilizados vinte LEDs de Alta Potência da OSRAM 720-GWPSL e um circuito com corrente constante em modo Off-line. A arquitetura proposta visa apresentar uma proposta de um driver de baixo custo e baixo consumo de corrente. É apresentado o estudo comparativo entre o protótipo deste dispositivo a partir dos gráficos obtidos com os resultados de medições em laboratório.

Palavras-chave: Conversor CUK; Lâmpada LED; Modo Contínuo de Corrente; Modulação por Largura de Pulso (MLP).

Recebido em: 19/08/2015 Revisado em: 25/08/2015 Aprovado em: 05/09/2015
ABSTRACT - This paper presents a new architecture of a driver of high brightness LEDs, with low cost and high yield. Employs a CUK type converter in continuous mode with current compensation rate to feed the high-brightness LEDs through the grid, from $85 \mathrm{~V}$ to $265 \mathrm{~V}$, operating at high frequency switched Pulse Width Modulation (MLP) and connected to a series LC resonant filter for load feeding entry in protection against voltage surges. Twenty LEDs High Power OSRAM 720-GWPSL and a circuit with constant current in Offline mode were used. The proposed architecture aims to present a proposal for a lowcost and low current consumption driver. The comparative study between the prototype of this device from the graphs obtained from the results of laboratory measurements.

Keywords: CUK Converter; LED Lamp; Continous Current Mode; Pulse Width Modulation (PWM). 


\section{INTRODUÇÃO}

Conforme nota técnica DEA 03/11 da Empresa de Pesquisa Energética (EPE), que apresenta uma projeção da demanda de energia elétrica para os próximos 10 anos, horizonte de médio prazo 2011 a 2020 (EPE, 2011), as consequências da crise financeira internacional manifestaram-se intensamente no comportamento do setor elétrico brasileiro, com forte retração do consumo industrial de eletricidade e nos primeiros meses de 2015 e um aumento acentuado na tarifação da energia elétrica em todas as classes de consumidores. Diante deste cenário, é de suma importância o desenvolvimento e utilização de materiais e equipamentos elétricos mais eficientes e de
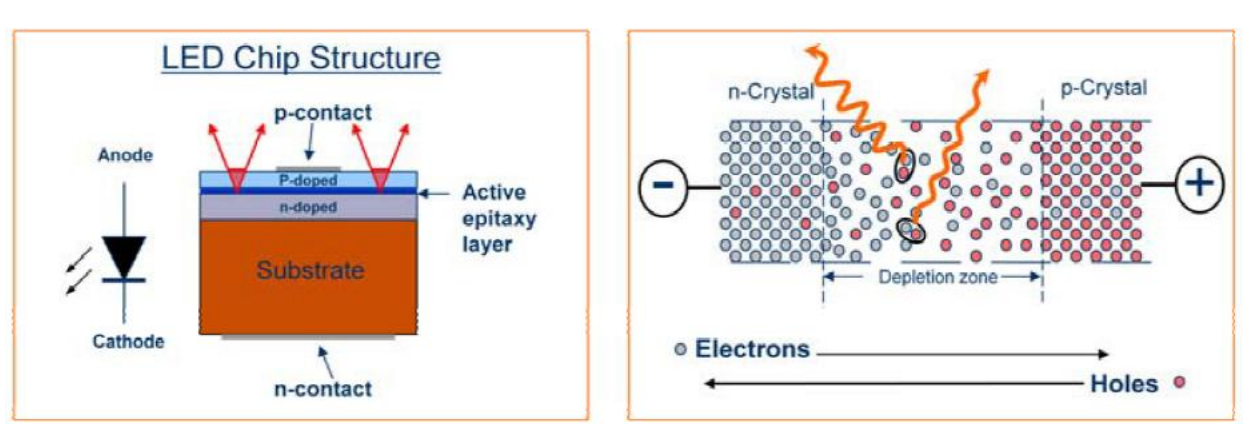

Figura 1. Representação da estrutura física e do comportamento de um LED (OSRAM Opto Semiconductors)

Na Figura 2 mostra a representação construtiva do LED empregado no protótipo. No interior da cavidade do LED é montada uma pastilha semicondutora sobre uma armação de terminais condutores, que são tecnologia de iluminação LED em grande escala. Os LEDs (diodos emissores de luz) são semicondutores constituídos por dois materiais diferentes que formam uma junção $\mathrm{PN}$, a qual permite o fluxo de corrente em apenas uma direção. Se a junção PN é polarizada diretamente, as lacunas na camada $\mathrm{P}$ e os elétrons da camada $\mathrm{N}$ movemse em direção à região de depleção (área de transição entre os materiais $\mathrm{P}$ e N). Perto dessa região, a recombinação de elétrons e lacunas gera energia que é liberada sob a forma de fótons de luz (BULLOUGH, 2003; U.S DEPARTAMENT PF ENERGY, 2009). Na Figura 1 é representada se forma simplificada a estrutura física do LED e do processo de emissão de luz (OSRAM, 2014).

de

baixo custo, para viabilização da utilização da 
para a Placa de Circuito Impresso (PCl). Um fio de ouro é utilizado para ligação do lado superior da pastilha para os terminais condutores laterais anodo (A) e catodo (C). A cavidade do invólucro é preenchida com resina epóxi e serve como refletor para extração da quantidade máxima de luz para o exterior do encapsulamento.

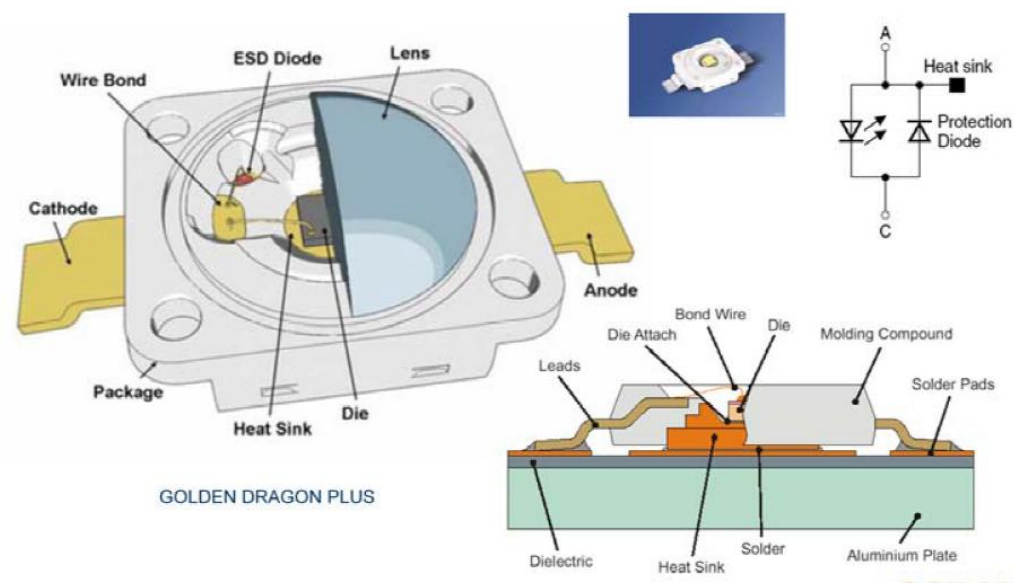

Figura 2. LED GW PSLRS1.EC de Alta Potência (OSRAM Opto Semiconductors)

No protótipo apresentado neste artigo são utilizados vinte LEDs, modelo GW PSLRS1.EC do fabricante OSRAM Opto Semiconductors. Os LEDs são alimentados por um driver constituído de quatro estágios. O primeiro estágio é composto por um filtro ressonante RLC (resistor-indutor-capacitor) e por uma proteção contra surto de tensão (varistor e fusistor), o segundo estágio por um retificador $A C-D C$, o terceiro estágio composto por um comutador primário com potência de $12 W$, frequência de chaveamento fixa em $42 \mathrm{kHz}$, modo contínuo de corrente e o último estágio é composto por um filtro de saída para redução a níveis aceitáveis de corrente de ondulação (MELLO,
2011), conforme a norma (IEC 61000-32:2014).

\section{METODOLOGIA}

O projeto da lâmpada LED do tipo bulbo começa com a escolha da potência de saída e com a especificação do fluxo luminoso. A maioria das lâmpadas incandescentes possuem potências entre 40 W e 100 W. Supondo que uma lâmpada de LED pode fazer o trabalho com uma eficiência $30 \%$ maior (devido à sua melhor eficácia luminosa e uma distribuição mais objetiva da iluminação sobre a área pretendida), a potência do conjunto de 9 LEDs poderia ser de cerca de 24 W. Estabelecida esta potência de $11 \mathrm{~W}$ almeja-se uma eficiência energética 
comparada com os modelos das incandescentes, mas com uma vida útil superior à dessas lâmpadas. Como os LEDs serão ligados em série, a tensão em seus terminais pode atingir baixos valores (operando com uma tensão típica de 6, 10V, assumindo LEDs de 1,2 W que apresentam tensão direta menores que $4 \mathrm{~V}$ entre seus terminais). Assumindo 20 LEDs associados em série, operando com 1,2 W cada, com tensão direta de aproximadamente $6,1 \mathrm{~V}$, será requerida uma corrente de 196 mA. Para essa corrente média, o valor de pico pode ser maior que $189 \mathrm{~mA}$ através dos LEDs empregando o conversor CUK. Embora a corrente média de $200 \mathrm{~mA}$ possa ser tolerada por alguns LEDs de $1,2 \mathrm{~W}$, eles teriam que operar acima da corrente limite de pico. Assim, neste caso, a potência do LED deve ser de $2 \mathrm{~W}$ ou $3 \mathrm{~W}$. Logo, para a implementação da lâmpada foi escolhido utilizar o LED GW PSLRS1.EC-LPLR-5U8X-1. Na Tabela 1 são exibidos seus principais parâmetros.

Tabela 1. Principais Características do LED GW PSLRS1.EC-LPLR-5U8X-1 (OSRAM)

\begin{tabular}{|c|c|c|c|}
\hline Parâmetro & Símbolo & Valor & Unidade \\
\hline Limite de Temperatura de Operação & $T_{\text {op }}$ & -40 a 110 & ${ }^{\circ} \mathrm{C}$ \\
\hline Temperatura de Junção & $T_{1}$ & 125 & ${ }^{\circ} \mathrm{C}$ \\
\hline Corrente Direta & $I_{d}$ & 10 a 200 & $\mathrm{~mA}$ \\
\hline Corrente Reversa & $\mathrm{I}_{R}$ & 20 & $\mathrm{~mA}$ \\
\hline Corrente de Surto & Is & 300 & $\mathrm{~mA}$ \\
\hline Ângulo de Visão em $50 \%$ & $2 \varphi$ & 120 & $\circ$ \\
\hline $\begin{array}{ll}\text { Tensão Direta } & (\min ) \\
& (\text { tip) } \\
& (\max ) \\
\end{array}$ & $\mathrm{V}_{d}$ & $\begin{array}{l}5.60 \\
6.10 \\
6.40 \\
\end{array}$ & $\begin{array}{l}\mathrm{V} \\
\mathrm{V} \\
\mathrm{V}\end{array}$ \\
\hline Temperatura Cor & $\mathrm{K}$ & 2700 & - \\
\hline $\begin{array}{ll}\text { Fluxo Luminoso } \mathrm{I}_{F}=150 \mathrm{~mA} & (\min ) \\
& (\max )\end{array}$ & ФV & $\begin{array}{l}112 \\
121\end{array}$ & $\mathrm{Im}$ \\
\hline Intensidade Luminosa & $I_{L}$ & 39,6 & $\mathrm{~cd}$ \\
\hline Resistência Equivalente & $\overline{\mathrm{R}_{E Q}}$ & 1,8 & $\bar{\Omega}$ \\
\hline
\end{tabular}

Utilizando os dados da Tabela 1, foram obtidas uma queda de tensão $\left(\mathrm{V}_{0}\right)$ de $24,3 \mathrm{~V}$ e uma resistência equivalente (Rs) de $16,2 \Omega$ para o conjunto de 9 LEDs. Assim, seria esperada uma tensão direta de 29,2 V. Neste caso, seria recomendado utilizar um transformador com tensão no secundário de $30 \mathrm{Vrms}$. Na equação 1 é apresentado o modelo elétrico para o LED utilizado: 


$$
V_{L E D}=\frac{\eta \cdot k \cdot T}{q} \cdot \ln \left(\frac{{ }^{I} L E D}{I_{S}}\right)+R s .{ }^{I} L E D \quad \text { Eq. (1) }
$$

Onde:

$V_{L E D}=$ Tensão Direta do LED em Volts (V);

$\eta=$ Fator de Idealidade;

$k=$ Constante de Boltzmann, 1.3805.10 ${ }^{-23} \quad\left(\mathrm{j} /{ }^{\circ} \mathrm{k}\right)$;

$\mathrm{T}=$ Temperatura em Kelvin $\left({ }^{\circ} k\right)$;

$q=$ Carga em Eletron-Volt, $1.602 .10^{-19}$ Coulomb (C);

${ }^{I} L E D=$ Corrente Direta do LED em Amperes (A);

$I_{S}=$ Corrente Direta de Saturação em Amperes (A).

Inicialmente realizou-se uma pesquisa bibliográfica afim de um embasamento teórico acerca dos conversores principalmente os conversores AC-CC, tema deste trabalho. Foi elaborado um estudo teórico da topologia do conversor AC-CC "CUK", operando em modo continuo de corrente. Buscou-se enfatizar a eficiência do protótipo frente às lâmpadas LED do tipo bulbo atuais, além de descrever o projeto e dimensionamento dos elementos constituinte da mesma. Para elucidar as etapas de projeto e dimensionamento foram feitos alguns estudos de caso. Confeccionaram-se tabelas para expor os dados resultantes desses estudos e propiciar a comparação dos mesmos.

\subsection{O CONVERSOR CUK NÃO ISOLADO}

O conversor chaveado desenvolvido para a lâmpada LED opera em frequência de $42 \mathrm{kHz}$, o que faz com que seu volume seja reduzido. O conversor é utilizado para a conversão $\mathrm{CC} / \mathrm{CC}$ que possui melhores características quanto a ruído emitido pelo conversor tanto na tensão de entrada (Ve) quanto a tensão de saída (Vs). A saída possui baixo ruído devido à característica não pulsante da corrente no indutor (BILLINGS, 1999). Para o conversor empregado, são adotados dois indutores, um na entrada e outro na saída, proporcionado uma baixa ondulação tanto na entrada quanto na saída, pois a corrente nos indutores é contínua.

A Figura 4 ilustra a diagrama elétrico do circuito proposto, cuja concepção seguiu o modelo de Ridley. 


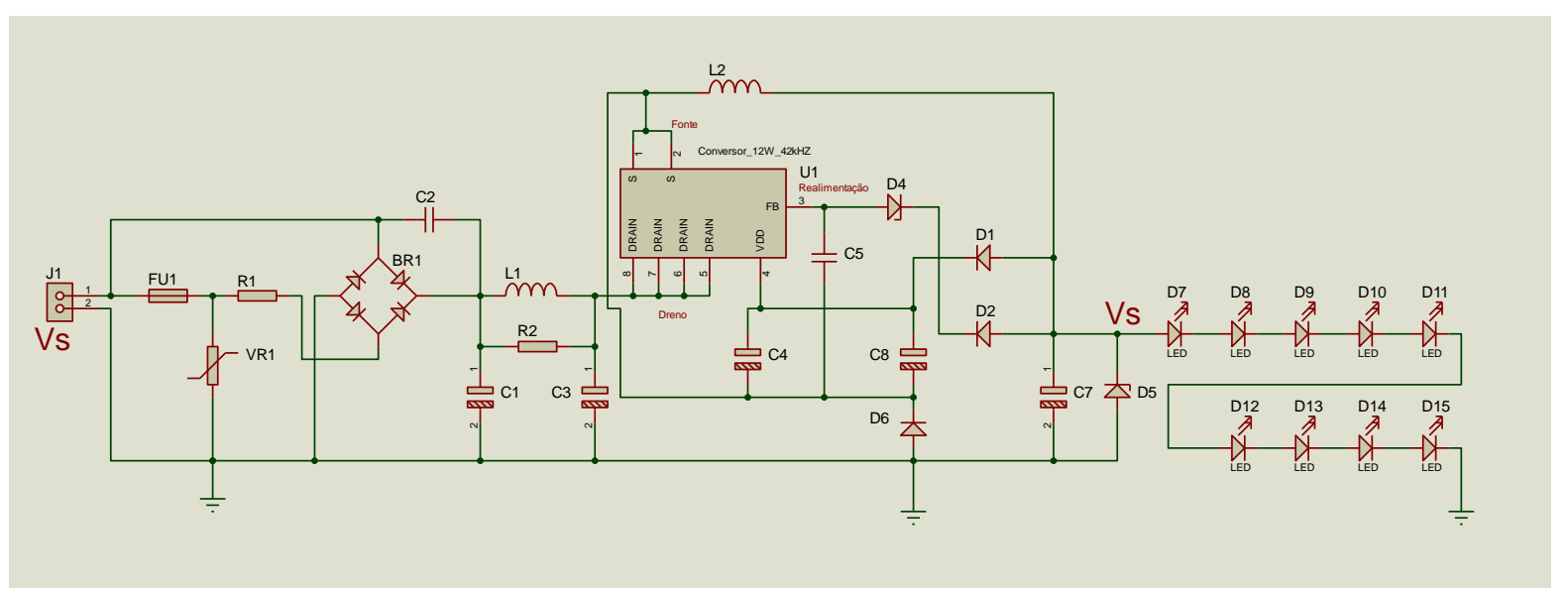

Figura 4. Diagrama elétrico do conversor CUK proposto

Em 1990, Dr. Raymond Ridley na Virginia Tech University (VPEC), apresentou um modelo de amostragem para o Modo de Controle de Corrente (MCC), modelando no estágio de potência do conversor uma forma polinomial de terceira ordem (KASSAKIAN; SCHLECHT; VERGHESE, 1991), que resultou em uma baixa frequência de um polo $\omega_{p} \mathrm{e}$ para duplo polo $\omega_{n}$, em um ciclo completo de chaveamento em frequência. A baixa frequência no polo $\omega^{\omega_{p}}$ resultou em uma baixa frequência de movimento em relação ao ciclo de trabalho, bem como para uma compensação externa do sinal em rampa quando presente, conforme demostrado na figura 5 .

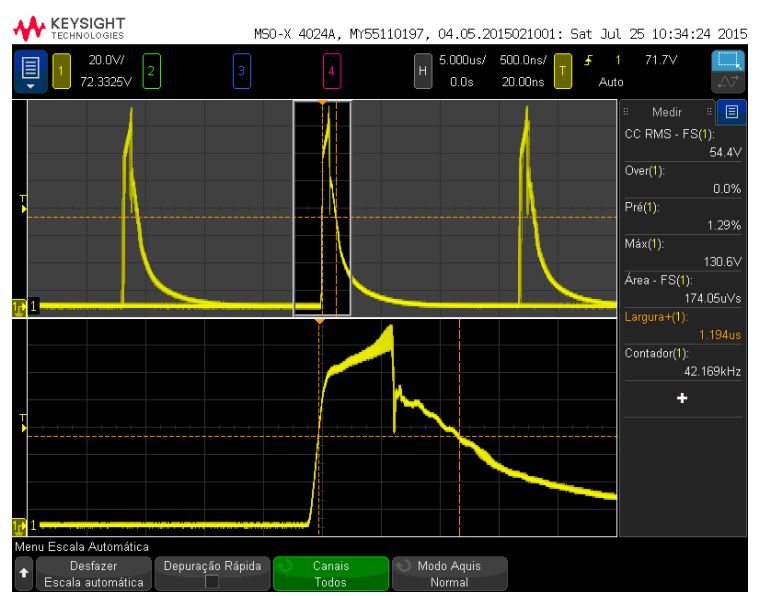

Figura 5. Frequência no indutor $L$ de saída para um polo $\omega_{p}$

A figura 6 apresenta a frequência medida no osciloscópio em laboratório para a ação do chaveamento no indutor de saída para uma corrente constante, atuando como um filtro de segunda ordem equivalente. 


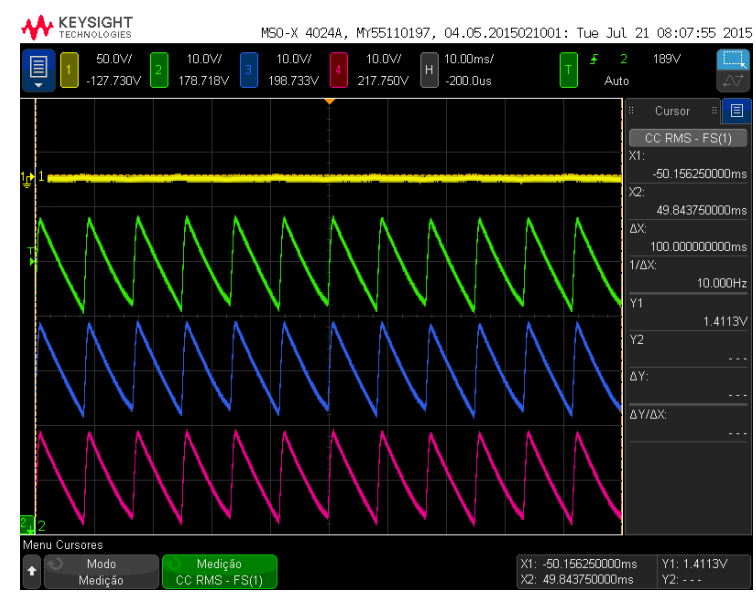

Figura 6. Frequência no indutor $L$ de entrada para um polo $\omega_{n}$

\section{RESULTADOS}

O conversor CA-CC tipo CUK de baixa frequência para um driver de lâmpada LED foi implementado no Laboratório de
Instrumentação e Eletroeletrônica (IEE), os resultados são apresentados na Tabela 2.

Tabela 2. Principais componentes eletrônicos utilizados na construção do driver.

\begin{tabular}{|c|c|}
\hline Parâmetro & Descrição \\
\hline Chaveamento por Largura do Pulso (MLP) & Circuito Integrado VIPEr22AS \\
\hline Indutor de Entrada & $1 \mu \mathrm{H}-100 \mathrm{~mA}$ \\
\hline Indutor de Saída & $1 \mu \mathrm{H}-200 \mathrm{~mA}$ \\
\hline Proteção Contra Surto na Entrada & Varistor EPCOS 250V \\
\hline Retificador de Onda Completo & Ponte Retificadora DF06 \\
\hline Proteção de Entrada & Fusível de $1 \mathrm{~A}$ - $250 \mathrm{~V}$ \\
\hline Proteção de Saída & Resistor $1.3 \Omega(\mathrm{Ohm})$ \\
\hline
\end{tabular}

\section{A figura 7 apresenta o protótipo}

montado em placa de circuito impresso padrão no laboratório. 


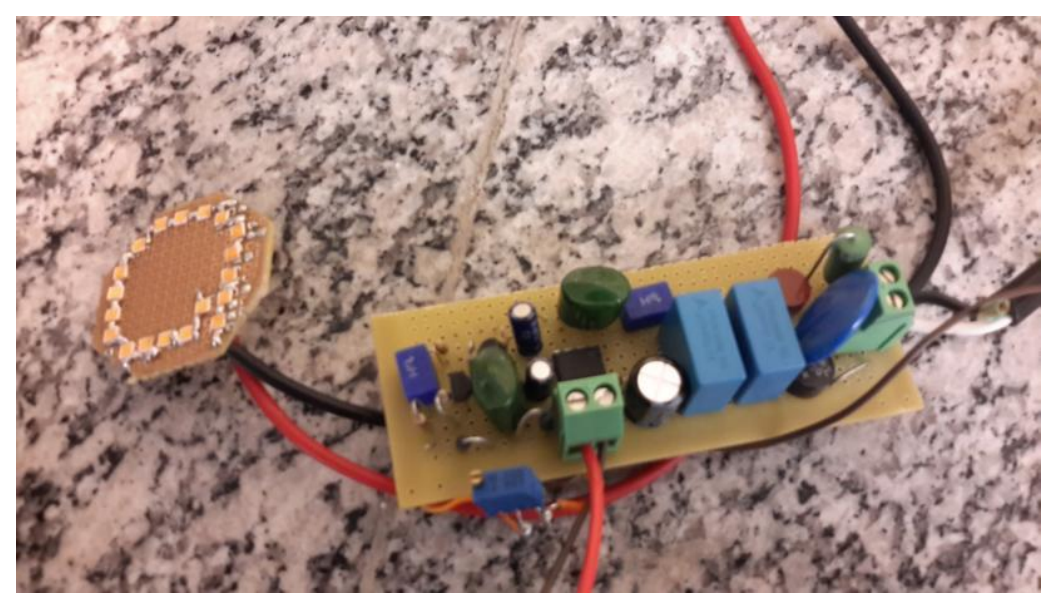

Figura 7. Protótipo montado em laboratório para com LED de alto brilho (luminância)

Conforme pode ser constatado na Figura 7 o protótipo foi construído em placa de circuito impresso padrão (matriz), com poucos componentes e baixo custo. Uma vantagem muito relevante no circuito desenvolvido é a ausência de transformador, pois a limitação da tensão é realizada através de capacitor e regulação pelo circuito integrado. Figura 8 mostra o teste de iluminância média do protótipo, cujo desempenho fotométrico do arranjo de LEDs foi comprovado através da medição da luminosidade medida por meio de um Luxímetro Digital. As medições foram realizadas com a fonte de luz a $100 \mathrm{~cm}$ do sensor do Luxímetro Digital para 6 posições diferentes. Foi obtida uma iluminância média de 843 Ix (Lux) para o conjunto de LEDs. Na figura 9 temos 0 conjunto de LEDs iluminando, onde é possível constatar a luminosidade uniforme.

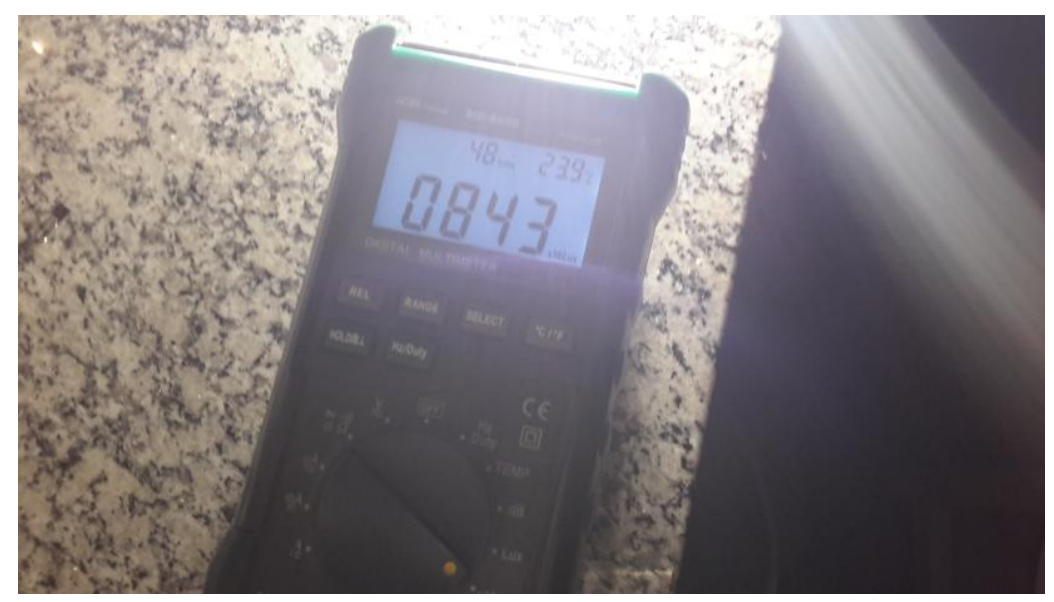

Figura 8. Teste de iluminância do protótipo com Luxímetro Digital 


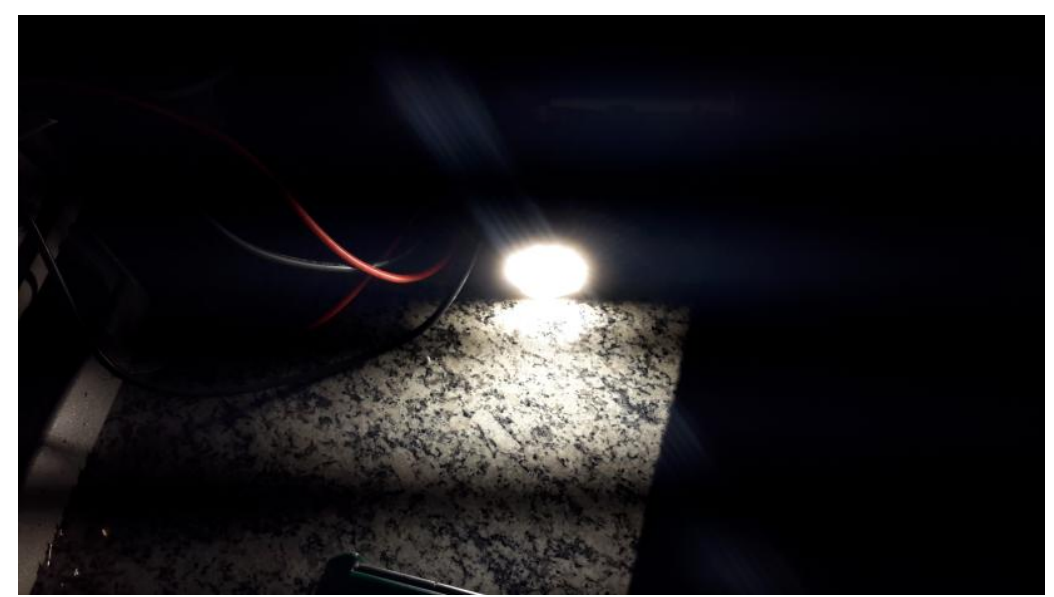

Figura 9. Arranjo de LEDs durante o teste de iluminância

\section{CONCLUSÃO}

Neste artigo foi apresentado o projeto de um driver para lâmpada LED de baixo custo e alta iluminância. Foram mostrados todas as etapas de desenvolvimento do projeto e os resultados experimentais obtidos com o protótipo. Foram realizados testes para avaliação da eficiência elétrica e fotométrica.

Nos testes foi constatada a alta eficiência apresentada pelo protótipo, tanto no consumo de energia em 189mA de corrente de carga, tanto na eficiência fotométrica com 843lx registrado como valor médio nas medições.

Como trabalho futuro o grupo de pesquisa do IEE, irá produzir uma lâmpada do tipo bulbo na impressora 3D e uma placa de circuito impresso industrializada com componentes eletrônicos montados sobre a superfície da placa.

\section{REFERÊNCIAS}

BILLINGS, K.H. Switchmode power supply handbook. New York: McGraw-Hill Professional, 1999.

BULLOUGH, J. D. Light Emitting Diode Lighting Systems. NLPIP - Lighting Answers. v. 7. Issue 3. May, 2003.

MELLO, L. Projeto de fontes chaveadasteoria e prática. São Paulo: Érica, 2011.

KASSAKIAN, J.G.; SCHLECHT, M.F.; VERGHESE, G.C. Principles of power electronics. Reading, USA: Addison-Wesley, 1991.

OSRAM Opto Semiconductors. Datasheet DURIS S 5 - Version 1.0. Regensburg: OSRAM, 2014.

U.S. DEPARTMENT OF ENERGY. Energy Efficiency and Renewable Energy. Thermal Management of White LEDs. November 2009.

http://www.epe.gov.br/mercado/Documents LS\%C3\%A9rie\%20Estudos\%20de\%20Energia/ 20110222 1.pdf. Acesso em 05/07/2015.

https://webstore.iec.ch/publication/4149. Acesso em 21/07/2015. 